\title{
High expression of ADAMTS5 is a potent marker for lymphatic invasion and lymph node metastasis in colorectal cancer
}

\author{
NAOTSUGU HARAGUCHI ${ }^{1}$, NOBUYOSHI OHARA ${ }^{1}$, JUN KOSEKI ${ }^{2}$, \\ HIDEKAZU TAKAHASHI ${ }^{1}$, JUNICHI NISHIMURA ${ }^{1}$, TAISHI HATA ${ }^{1}$, TSUNEKAZU MIZUSHIMA ${ }^{1}$, \\ HIROFUMI YAMAMOTO $^{1}$, HIDESHI ISHII ${ }^{2}$, YUICHIRO DOKI ${ }^{1}$ and MASAKI MORI ${ }^{1}$ \\ Departments of ${ }^{1}$ Gastroenterological Surgery and ${ }^{2}$ Cancer Profiling Discovery, \\ Graduate School of Medicine, Osaka University, Osaka 565-0871, Japan
}

Received January 19, 2016; Accepted September 29, 2016

DOI: $10.3892 / \mathrm{mco} .2016 .1088$

\begin{abstract}
Members of the ADAMTS family contain propeptide, metalloproteinase and disintegrin domains and serve key roles for cancer cell proliferation, progression and metastasis. Although overexpression of ADAMTS5 has been reported in glioblastoma, and head and neck cancer, little has been demonstrated in colorectal cancer types. The present study aimed to clarify the significance of ADAMTS5 for clinicopathological factors and prognosis in colorectal cancer. The mRNA expression of ADAMTS5 was measured in 143 colorectal cancer specimens. ADAMTS5 expression was increased as the pathological stage increased. The expression of ADAMTS5 in stage III-IV colorectal cancer was significantly greater compared with that of stage 0 -II colorectal cancer $(\mathrm{P}=0.0003)$. The median expression of ADAMTS5 was used to divide the ADAMTS5 higher expressing group and the ADAMTS5 lower expressing group to assess the correlation of ADAMTS5 expression with clinicopathological factors and prognosis. The proportions of lymphatic invasion and lymph node metastasis were significantly greater in the ADAMTS5 higher expressing group $(\mathrm{P}=0.0214$ and $\mathrm{P}=0.0289$ respectively). Overall survival and disease-free survival were assessed by the Kaplan-Meier curve with calculation of significance by the log-rank test; however, no significant difference was observed between the ADAMTS5 higher expressing group and the ADAMTS5 lower expressing group $(\mathrm{P}=0.7490$ and $\mathrm{P}=0.9455$, respectively). The present study confirmed high expression of ADAMTS5 as a potent marker for lymphatic invasion and lymphnode metastasis in colorectal cancer. To clarify the function of ADAMTS5 in colorectal cancer, further molecular investigations are required.
\end{abstract}

Correspondence to: Dr Naotsugu Haraguchi, Department of Gastroenterological Surgery, Graduate School of Medicine, Osaka University, Suita Yamadaoka 2-2-E2, Osaka 565-0871, Japan

E-mail: nharaguchi@gesurg.med.osaka-u.ac.jp

Key words: ADAMTS5, colorectal cancer, lymphatic invasion, lymph node metastasis

\section{Introduction}

Cancer of the gastrointestinal system are a leading cause of mortality worldwide and are more prevalent compared with breast cancer and brain tumors. In Japan, cancer of seven gastrointestinal system sites, including the esophagus, stomach, colorectum, liver and pancreas, are listed among the top 10 causes of cancer-associated mortality. Colorectal cancer (CRC) is the fourth leading cause of mortality worldwide, and there are 1.2 million novel CRC cases and $\sim 608,000$ mortalities occurred in 2008 (1). In 2012, an estimated 1.4 million novel CRC cases and 693,900 mortalities, and the incidence rates are increasing in numerous countries (2-4). Despite rising incidence of CRC, mortality rates of CRC are decreasing worldwide, likely due to screening and improved treatment $(5,6)$. In CRC, prognosis of patients have been drastically improving, particularly with the combination of chemotherapeutic agents and development of molecular target drugs, including anti-epidermal growth factor receptor (EGFR) antibody, anti-vascular endothelial growth factor (VEGF) antibody and multikinase inhibitor (7-11). In previous years, screenings of cancer-associated molecules have been eagerly performed to identify the novel molecular targets and development of novel anticancer agents.

The present study focused on the expression of ADAMTS5 in colorectal cancer. ADAMTS family members contain thrombospondin motifs, cysteine-rich and spacer domains in addition to propeptide, metalloproteinase and disintegrin domains (12). The matrix metalloproteinases (MMPs) serve key roles for cancer cell proliferation, progression and metastasis in numerous human cancer types via their activity of degradation of the extracellular matrix (13). Although overexpression of ADAMTS5 has been reported in glioblastoma (14-16), and head and neck cancer (17), the expression and the roles of ADAMTS5 remain to be understood in colorectal cancer types. The present study measured the expression of ADAMTS5, and clarified the roles of ADAMTS5 by the assessment of mRNA expression and clinicopathological factors in colorectal cancer.

\section{Materials and methods}

Patients and specimens. Specimens of primary colorectal cancer samples were obtained from 143 patients with colorectal 
cancer who underwent surgery between March 2003 and June 2006. All of the specimens were obtained with approval with informed consent from the patients, and were stored with anonymized clinicopathological data at Osaka University (Osaka, Japan). Retrospective analysis using stored tumor specimens was performed under the acceptance of the Research Ethics Board of Osaka University. The present study utilized cDNAs of colorectal cancer specimens to evaluate the expression of ADAMTS5.

Semi-quantitative polymerase chain reaction (PCR). Using stored cDNAs from colorectal cancer specimens, the present study evaluated the expression of ADAMTS5. The PCR was performed using the Light Cycler (Roche Diagnostics Deutschland GmbH, Mannheim, Germany). The primers used were as follows: ADAMTS5, forward: 5'-GCTACTGCACAG GGAAGAGG-3' and reverse: 5'-TGCATATTTGGGAACCCA TT-3'; GAPDH (internal control), forward 5'-CAACTACAT GGTTTACATGTTC-3' and reverse: 5'-GCCAGTGGACTC CACGAC-3'. The amplification protocol consisted of 55 cycles of denaturation at $95^{\circ} \mathrm{C}$ for $5 \mathrm{sec}$, annealing at $60^{\circ} \mathrm{C}$ for $5 \mathrm{sec}$ and extension at $72^{\circ} \mathrm{C}$ for $30 \mathrm{sec}$.

Definition of tumor stage. Tumor stages were defined according to the tumor-node-metastasis (TNM) Classification of Malignant Tumours 7th Edition [Union Internationale Contre le Cancer (UICC)].

Statistical analysis. The present study divided cases for analysis based on the expression of ADAMTS5 (Low or High). The median score of ADAMTS5 expression was used to define the higher ADAMTS5 expression group (High group) and the lower expression group (Low group). Fisher's exact test was used to compare the differences between the ADAMTS5 High and Low groups. The present study assessed the overall survival (OS) and disease-free survival (DFS) of these two groups using the Kaplan-Meier method. The log-rank test calculates the significance of any differences. Cases of non-curative resection were excluded in the DFS analyses. Univariate and multivariate analyses for OS or DFS were performed to evaluate the independent prognostic factors using Cox proportional hazards model. All statistic analyses were performed using JMP 11.0.0 software (SAS Institute, Cary, NC, USA). P<0.05 was considered to indicate a statistically significant difference.

\section{Results}

Expression of ADAMTS5 in colorectal cancer. Expression of ADAMTS5 was assessed in the 143 colorectal cancer specimens. ADAMTS5 expression was relatively low; however, was identified in all of the 143 specimens. The expression of ADAMTS5 was increased as the TNM stage increased $(\mathrm{P}=0.0063$; Fig. 1A). When TNM stage was divided into the two groups, stage 0 -II and stage III-IV, expression of ADAMTS5 was significantly higher in the TNM stage III-IV compared with the TNM stage $0-\mathrm{II}(\mathrm{P}=0.0003$; Fig. 1B).

Expression of ADAMTS5 and clinicopathological factors. To assess the association between the expression of ADAMTS5 and clinicopathological factors and prognosis, the present study divided patients based on the median score of ADAMTS5 expression into two groups: ADAMTS5 high and low expression groups. The ADAMTS5 low group included 72 patients and the ADAMTS5 high group included 71 patients $(\mathrm{P}=0.1533)$. The ADAMTS5 low group included 52 males and 19 females, and the ADAMTS5 high group involved 43 males and 28 females. No significant differences in the histological type $(\mathrm{P}=1.000)$, venous invasion $(\mathrm{P}=0.7348)$ and depth of tumor invasion $(\mathrm{P}=0.4205)$ was observed between the ADAMTS5 low group and the ADAMTS5 high group (Table I). In the analysis of clinicopathological factors, the proportions of lymphatic invasion $(\mathrm{P}=0.0214)$ and lymph node metastasis $(\mathrm{P}=0.0289)$ were significantly greater in the ADAMTS5 high group compared with the ADAMTS5 low group. Although the TNM stage was potentially greater in the ADAMTS5 high group compared with the ADAMTS5 low group ( $\mathrm{P}=0.0896)$, the proportion of the distant metastasis was not significantly different between these two groups $(\mathrm{P}=0.1675)$.

Expression of ADAMTS5 and clinical outcome. Correlation of the expression of ADAMTS5 with clinical outcome was assessed by the comparison of the ADAMTS5 high group and the ADAMTS5 low group. Univariate and multivariate analyses indicated that lymphatic invasion, venous invasion and lymph node metastasis were independent prognostic factors for OS (Table II). The expression of ADAMTS5 was not characterized as an independent prognostic factor for OS $(\mathrm{P}=0.7490)$. The OS and DFS were assessed by the Kaplan-Meier method, using the log-rank test. The Kaplan-Meier curves revealed no significant differences in the OS between the ADAMTS5 low group and the ADAMTS5 high group ( $\mathrm{P}=0.7485$; Fig. 2A).

Next, the present study assessed the correlation between ADAMTS5 expression and DFS in the 125 patients; 18 patients from the 143 patients were excluded from this analysis due to non-curative surgery. Adjuvant chemotherapy was performed on the 16 patients $(25.4 \%)$ of the ADAMTS5 low group and on the 24 patients $(38.71 \%)$ of the ADAMTS5 high group. Relapse was observed in the 27 patients; 4 patients $(22.2 \%)$ in the ADAMTS5 low group and 13 patients (21.0\%) in the ADAMTS5 high group, respectively. No significant correlation was observed between the expression of ADAMTS5 and the proportion of recurrence (Table III; $\mathrm{P}=1.000$ ). No significant correlation was observed between ADAMTS5 expression and DFS, based on the Kaplan-Meier curve with log-rank test ( $\mathrm{P}=0.9455$; Fig. 2B). Univariate analyses revealed no significance of the ADAMTS5 expression on DFS (hazard risk=1.026; $95 \%$ confidence interval=0.4799-2.211; $\mathrm{P}=0.9455$ ).

\section{Discussion}

ADAMTS5, also termed aggrecanase-2 due to aggrecandegrading activity, is a member of the $\operatorname{ADAM}$ superfamily $(18,19)$. The proteoglycanase activity of ADAMTS5, known as an member of extracellular matrix (ECM) degrading enzymes, shows proteolytic activity toward the hyalectan group of chondroitin sulphate proteoglycans (CSPGs), which comprise aggrecan, versican, brevican and neurocan (20). Although ADAMTS5 have been implicated in various cellular events, including cleavage of proteoglycans, ECM degradation, inhibition of angiogenesis and embryonic morphogenesis (21), their 
Table I. Baseline characteristics of clinicopathological factors.

\begin{tabular}{|c|c|c|c|}
\hline \multirow[b]{2}{*}{ Characteristic } & \multicolumn{2}{|c|}{ ADAMTS5 } & \multirow[b]{2}{*}{ P-value } \\
\hline & Low group $(n=72)$ & High group $(n=71)$ & \\
\hline Gender & & & 0.1533 \\
\hline Male, n (\%) & $52(73.24)$ & $43(60.56)$ & \\
\hline Female, n (\%) & $19(26.76)$ & $28(39.44)$ & \\
\hline Histologic type & & & 1.000 \\
\hline tub1-tub2,n (\%) & $69(95.83)$ & $68(95.77)$ & \\
\hline por-sig, n $(\%)$ & $3(4.17)$ & $3(4.23)$ & \\
\hline Lymphatic invasion & & & 0.0214 \\
\hline Positive, n (\%) & $47(65.28)$ & $59(83.10)$ & \\
\hline Negative, n (\%) & $25(34.72)$ & $12(16.90)$ & \\
\hline Venous invasion & & & 0.7348 \\
\hline Positive, n (\%) & $41(56.94)$ & $43(60.56)$ & \\
\hline Negative, n $(\%)$ & $31(43.06)$ & $28(39.44)$ & \\
\hline Depth of tumor invasion & & & 0.4205 \\
\hline $\mathrm{T} 0 / \mathrm{T} 1 / \mathrm{T} 2$ & $6 / 5 / 7$ & $2 / 1 / 10$ & \\
\hline $\mathrm{T} 3$ & 25 & 28 & \\
\hline $\mathrm{T} 4 \mathrm{a}$ & 22 & 25 & \\
\hline $\mathrm{T} 4 \mathrm{~b}$ & 7 & 3 & \\
\hline T0-2/T3-4 & $18 / 54$ & $13 / 56$ & \\
\hline Lymph node metastasis & & & 0.0289 \\
\hline Positive, n (\%) & $25(34.72)$ & $38(53.52)$ & \\
\hline Negative, n (\%) & $47(65.28)$ & $33(46.48)$ & \\
\hline UICC stage & & & 0.0896 \\
\hline 0/I/II/III/IV & $5 / 12 / 29 / 17 / 9$ & $2 / 9 / 18 / 27 / 15$ & \\
\hline Distant metastasis & & & 0.1675 \\
\hline Positive & 9 & 15 & \\
\hline Negative & 63 & 56 & \\
\hline
\end{tabular}

UICC, the union internationale contre le cancer.

Table II. Univariate and multivariate analysis associated with overall survival.

\begin{tabular}{|c|c|c|c|c|c|c|c|}
\hline \multirow[b]{2}{*}{ Characteristic } & \multirow[b]{2}{*}{$\mathrm{n}$} & \multicolumn{3}{|c|}{ Univariate analysis } & \multicolumn{3}{|c|}{ Multivariate analysis } \\
\hline & & HR & $95 \% \mathrm{CI}$ & P-value & HR & $95 \% \mathrm{CI}$ & P-value \\
\hline Gender (male/female) & $95 / 47$ & 1.1 & $0.516-2.542$ & 0.8101 & - & - & - \\
\hline ADAMTS5 expression (high/low) & $71 / 72$ & 0.887 & $0.422-1.852$ & 0.749 & - & - & - \\
\hline Pathological type (por-sig/tub1-2) & $6 / 137$ & 5.301 & $1.252-15.34$ & 0.0273 & 1.79 & $0.418-5.314$ & 0.3851 \\
\hline Lymphatic invasion (+/-) & $106 / 37$ & - & - & 0.0001 & - & - & 0.0158 \\
\hline Venous invasion $(+/-)$ & $84 / 59$ & 10.97 & $3.290-68.04$ & 0.0001 & 5.996 & $1.770-37.40$ & 0.0019 \\
\hline pT (T3-4/T0-2) & $110 / 31$ & 9.084 & $1.939-161.9$ & 0.0017 & 2.895 & $0.592-52.31$ & 0.2262 \\
\hline $\mathrm{pN}(+/-)$ & $63 / 80$ & 6.371 & $2.750-17.31$ & 0.0001 & 2.548 & $1.066-7.089$ & 0.0347 \\
\hline
\end{tabular}

$\mathrm{HR}$, hazard ratio; $\mathrm{CI}$, confidence interval; $\mathrm{T}$, tumor stage; $\mathrm{N}$, node.

roles in cancer remain to be established. Upregulated expression of ADAMTS5 has been reported in proliferating glioblastoma cells (14), and ADAMTS5 cleaves brevican and serves an important role in glioma cell invasion (15). In head and neck cancer, mRNA expression of ADAMTS5 has been reported to overexpress in metastatic foci (17). By contrast, downregulation 
Table III. Expression of ADMATS5 and recurrence.

\section{ADAMTS5}

Characteristic

Low group $(n=63)$

High group $(n=62)$

P-value

Adjuvant chemotherapy

Yes

$16(25.40 \%)$

0.1277

No

$47(74.60 \%)$

$24(38.71 \%)$

$38(61.29 \%)$

Recurrence

Yes

$14(22.22 \%)$

$13(20.97 \%)$

No

$49(77.78 \%)$

$49(79.03 \%)$
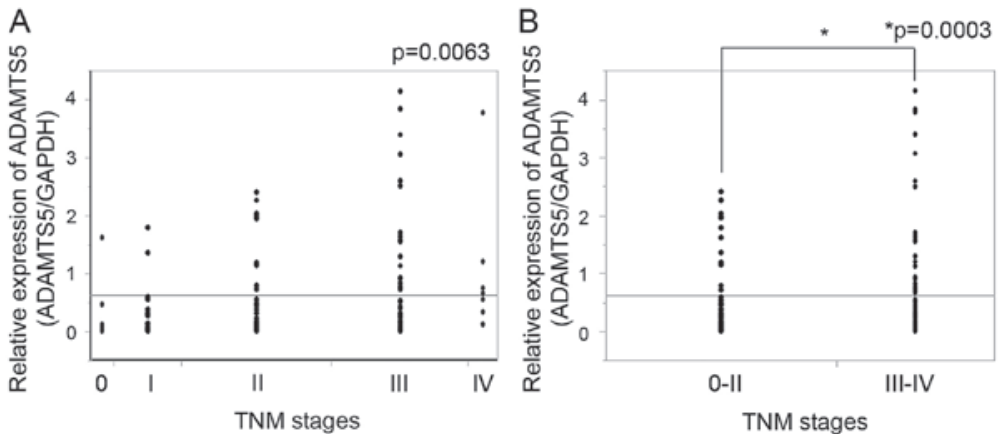

Figure 1. ADAMTS5 expression and TNM stages. (A) ADAMTS5 expression in stage 0, I, II, III and IV colorectal cancer specimens and (B) ADAMTS5 expression of stage 0-II and stage III-IV colorectal cancer specimens were determined. TNM, tumor-node-metastasis.
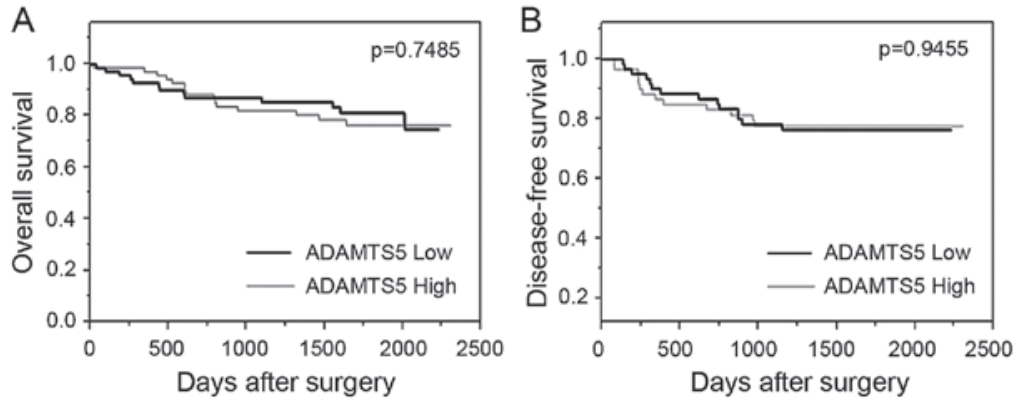

Figure 2. Kaplan-Meier curves revealed long-term survival in colorectal cancer patients, stratified to the expression status of ADAMTS5. The (A) overall survival and (B) disease-free survival curves were calculated for the ADAMTS5 low expressing group and the ADAMTS5 high expressing group.
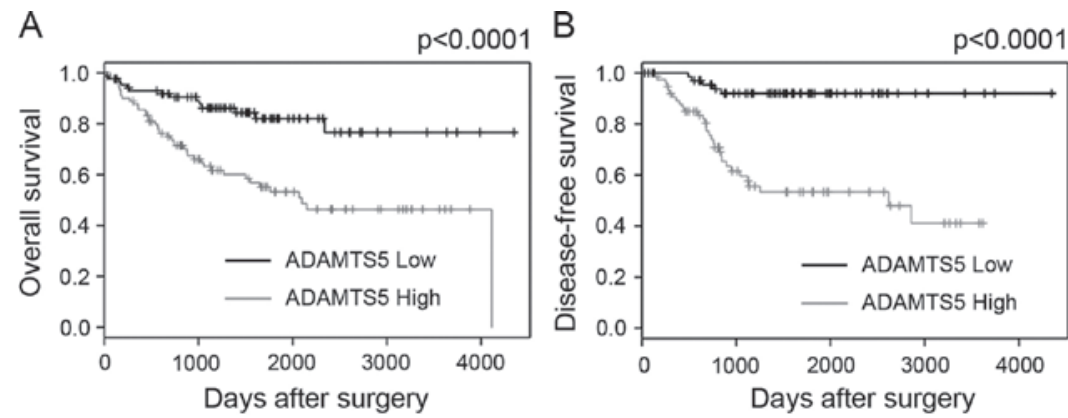

Figure 3. Kaplan-Meier curves from the PrognoScan database. The (A) overall survival of 177 patients and the (B) disease-free survival of 145 patients with colon cancer. The median value of intensity of microarray probes for ADAMTS5 was used to divide the ADAMTS5 high group and the ADAMTS5 low group.

of ADAMTS5 expression has been reported in prostate cancer and TGF- $\beta$ exposed prostatic stromal cells (22). In a previous study of the breast, while ADAMTS5 was expressed predominantly in myoepithelial cells, ADAMTS5 gene expression was 
downregulated in cancer tissues (23). In the present study, ADAMTS5 expression in colorectal cancer was significantly correlated with the lymphatic invasion and lymph node metastasis. Expression of ADAMTS5 increased according to TNM stage; stage III-IV colorectal cancer specimens expressed higher level of ADAMTS5 compared with stage 0-II. Considering that lymph node metastasis status divides stage III from stage 0-II, and that ADAMTS5 expression increase markedly in stage III, ADAMTS5 expression is potentially available as a marker of lymph node metastasis in colorectal cancer.

The present study failed to identify ADAMTS5 as an independent prognostic factor of colorectal cancer. Univariate analysis and the Kaplan-Meier curves revealed no significant impact of ADAMTS5 as a prognostic factor. To confirm these results, microarray data of colorectal cancer specimens listed on PrognoScan (http://www.abren.net/PrognoScan/) were used (24). The microarray data sets (http://www.ncbi.nlm.nih. gov/geo/query/acc.cgi?acc=GSE17536) used in the previous report of gene expression profile analysis (25) was used for analysis. The median value of intensity of microarray probes of ADAMTS5 was used for the ADAMTS5 low expressing group and the ADAMTS5 high expressing group. OS and DSF of the ADAMTS5 high expressing patients were significantly poorer compared with those of the ADAMTS5 low expressing patients $(\mathrm{P}<0.0001$ in OS and DSF) (Fig. 3). Although the factor that causes such large differences cannot be fully clarified, difference of sequence between microarray probe and PCR probe can be listed as one of the reasons. Difference of post-operative treatment and/or difference of concept of lymphadenectomy may potentially affect these differences. Expression and roles of ADAMTS5 remain to be determined in colorectal cancer (26). Further studies, including molecular based analysis and protein analysis, are required.

\section{Acknowledgements}

The authors would like to thank Ms. Yurika Nakamura at Departments of Gastroenterological Surgery, Graduate School of Medicine, Osaka University for excellent technical assistance.

\section{References}

1. Ferlay J, Shin HR, Bray F, Forman D, Mathers C and Parkin DM: Estimates of worldwide burden of cancer in 2008: GLOBOCAN 2008. Int J Cancer 127: 2893-2917, 2010.

2. Torre LA, Siegel RL, Ward EM and Jemal A: Global cancer incidence and mortality rates and trends-an update. Cancer Epidemiol Biomarkers Prev 25: 16-27, 2015.

3. Bray F: The evolving scale and profile of cancer worldwide: Much ado about everything. Cancer Epidemiol Biomarkers Prev 25: 3-5, 2016

4. Cunningham D, Atkin W, Lenz HJ, Lynch HT, Minsky B, Nordlinger B and Starling N: Colorectal cancer. Lancet 375 1030-1047, 2010

5. Bosetti C, Levi F, Rosato V, Bertuccio P, Lucchini F, Negri E and La Vecchia C: Recent trends in colorectal cancer mortality in Europe. Int J Cancer 129: 180-191, 2011.

6. Edwards BK, Ward E, Kohler BA, Eheman C, Zauber AG, Anderson RN, Jemal A, Schymura MJ, Lansdorp-Vogelaar I, Seeff LC, et al: Annual report to the nation on the status of cancer, 1975-2006, featuring colorectal cancer trends and impact of interventions (risk factors, screening, and treatment) to reduce future rates. Cancer 116: 544-573, 2010.

7. Jonker DJ, O'Callaghan CJ, Karapetis CS, Zalcberg JR, Tu D, Au HJ, Berry SR, Krahn M, Price T, Simes RJ, et al: Cetuximab for the treatment of colorectal cancer. N Engl J Med 357: 2040-2048, 2007.
8. Karapetis CS, Khambata-Ford S, Jonker DJ, O'Callaghan CJ, Tu D, Tebbutt NC, Simes RJ, Chalchal H, Shapiro JD, Robitaille S, et al: K-ras mutations and benefit from cetuximab in advanced colorectal cancer. N Engl J Med 359: 1757-1765, 2008.

9. Van Cutsem E, Peeters M, Siena S, Humblet Y, Hendlisz A, Neyns B, Canon JL, Van Laethem JL, Maurel J, Richardson G, et al: Open-label phase III trial of panitumumab plus best supportive care compared with best supportive care alone in patients with chemotherapy-refractory metastatic colorectal cancer. J Clin Oncol 25: 1658-1664, 2007.

10. Hurwitz H, Fehrenbacher L, Novotny W, Cartwright T, Hainsworth J, Heim W, Berlin J, Baron A, Griffing S, Holmgren E, et al: Bevacizumab plus irinotecan, fluorouracil, and leucovorin for metastatic colorectal cancer. N Engl J Med 350: 2335-2342, 2004.

11. Grothey A, Van Cutsem E, Sobrero A, Siena S, Falcone A, Ychou M, Humblet Y, Bouché O, Mineur L, Barone C, et al: Regorafenib monotherapy for previously treated metastatic colorectal cancer (CORRECT): An international, multicentre, randomised, placebo-controlled, phase 3 trial. Lancet 381: 303-312, 2013.

12. Mochizuki S and Okada Y: ADAMs in cancer cell proliferation and progression. Cancer Sci 98: 621-628, 2007.

13. Egeblad M and Werb Z: New functions for the matrix metalloproteinases in cancer progression. Nat Rev Cancer 2: 161-174, 2002.

14. Held-Feindt J, Paredes EB, Blömer U, Seidenbecher C, Stark AM, Mehdorn HM and Mentlein R: Matrix-degrading proteases ADAMTS4 and ADAMTS5 (disintegrins and metalloproteinases with thrombospondin motifs 4 and 5) are expressed in human glioblastomas. Int J Cancer 118: 55-61, 2006.

15. Nakada M, Miyamori H, Kita D, Takahashi T, Yamashita J, Sato H, Miura R, Yamaguchi Y and Okada Y: Human glioblastomas overexpress ADAMTS-5 that degrades brevican. Acta Neuropathol 110: 239-246, 2005.

16. Viapiano MS, Hockfield S and Matthews RT: BEHAB/brevican requires ADAMTS-mediated proteolytic cleavage to promote glioma invasion. J Neurooncol 88: 261-272, 2008.

17. Demircan K, Gunduz E, Gunduz M, Beder LB, Hirohata S, Nagatsuka H, Cengiz B, Cilek MZ, Yamanaka N, Shimizu K and Ninomiya Y: Increased mRNA expression of ADAMTS metalloproteinases in metastatic foci of head and neck cancer. Head Neck 31: 793-801, 2009.

18. Hurskainen TL, Hirohata S, Seldin MF and Apte SS: ADAM-TS5, ADAM-TS6 and ADAM-TS7, novel members of a new family of zinc metalloproteases. General features and genomic distribution of the ADAM-TS family. J Biol Chem 274: 25555-25563, 1999.

19. Apte SS: A disintegrin-like and metalloprotease (reprolysin-type) with thrombospondin type 1 motif (ADAMTS) superfamily: Functions and mechanisms. J Biol Chem 284: 31493-31497, 2009.

20. Schaefer L and Schaefer RM: Proteoglycans: From structural compounds to signaling molecules. Cell Tissue Res 339: 237-246, 2010.

21. Kintakas $\mathrm{C}$ and McCulloch DR: Emerging roles for ADAMTS5 during development and disease. Matrix Biol 30: 311-317, 2011.

22. Cross NA, Chandrasekharan S, Jokonya N, Fowles A, Hamdy FC, Buttle DJ and Eaton CL: The expression and regulation of ADAMTS-1, $-4,-5,-9$ and -15 and TIMP-3 by TGFbeta1 in prostate cells: Relevance to the accumulation of versican. Prostate 63: 269-275, 2005.

23. Porter S, Scott SD, Sassoon EM, Williams MR, Jones JL, Girling AC, Ball RY and Edwards DR: Dysregulated expression of adamalysin-thrombospondin genes in human breast carcinoma. Clin Cancer Res 10: 2429-2440, 2004.

24. Mizuno H, Kitada K, Nakai K and Sarai A: PrognoScan: A new database for meta-analysis of the prognostic value of genes. BMC Med Genomics 2: 18, 2009.

25. Smith JJ, Deane NG, Wu F, Merchant NB, Zhang B, Jiang A, Lu P, Johnson JC, Schmidt C, Bailey CE, et al: Experimentally derived metastasis gene expression profile predicts recurrence and death in patients with colon cancer. Gastroenterology 138: 958-968, 2010.

26. Kim YH, Lee HC, Kim SY, Yeom YI, Ryu KJ, Min BH, Kim DH, Son HJ, Rhee PL, Kim JJ, et al: Epigenomic analysis of aberrantly methylated genes in colorectal cancer identifies genes commonly affected by epigenetic alterations. Ann Surg Oncol 18: 2338-2347, 2011. 